\title{
Isokinetic studies for detection of functional disorders in patients with unilateral shoulder impingement syndrome
}

\author{
Katarzyna Połyniak', Przemysław Lisiński', Juliusz Huber², Leszek Romanowski ${ }^{3}$ \\ ${ }^{1}$ Department of Rehabilitation, Poznan University of Medical Sciences, Poland \\ 2 Department of Pathophysiology of Locomotor Organs, Poznan University of Medical Sciences, Poland \\ ${ }^{3}$ Department of Orthopaedics, Traumatology and Hand Surgery, Poznan University of Medical Sciences, Poland
}

\begin{abstract}
Introduction. The isokinetic assessment allows objective evaluation of muscle strength in patients considered for surgery in cases of rotator cuff injury.

Aim. The goal of this study was to define functional disorders of shoulder joint in patients with rotator cuff injury.

Material and methods. The examination was conducted in two groups, ten patients each. One group consisted of subjects with rotator cuff injury while the other was a healthy control group. Isokinetic test was performed with use of Biodex System 4 Pro Device. The following parameters were evaluated: peak torque, peak torque/body weight (peak TQ/BW), total work, average power, range of movement (ROM) and peak torque ratio of external to internal rotators (ERPT/IRPT ratio). Studies of Shoulder Pain and Disability Index (SPADI) scale supplemented clinical evaluation.

Results. Examination indicated a significant deficit of muscle strength during external rotation and ROM limitation only on symptomatic side in shoulder impingement group. Alteration between agonistic and antagonistic muscles strength for $240 \% \mathrm{~s}$ was found. Significant differences between involved and uninvolved shoulders during pain and disability tests were detected. There was no correlation between result of isokinetic and SPADI tests.

Conclusions. Patients after injury of rotator cuff present functional disorders that occur mainly during external rotation in isokinetic evaluation.
\end{abstract}

Keywords: shoulder impingement syndrome, rotator cuff, isokinetic dynamometry, shoulder pain.

\section{Introduction}

Rotator cuff injury combined with shoulder impingement syndrome is a common pathology of shoulder joint. Frequency of prevalence increases with age. After conducting USG tests it was observed that $13 \%$ of the population in their fifties, $20 \%$ in their sixties and $31 \%$ in their seventies suffer from this pathology [1]. Symptoms of rotator cuff disease include pain, muscles weakness, decreased range of motion, and as a result the impaired functionality of shoulder joint in daily activities [2-4].

The isokinetic assessment becomes more and more common in orthopedic practice. It allows for the objec- tive evaluation of muscle strength in various velocities and different positions of the subject's body. The results of evaluation make possible to examine a lot of different parameters. However, most studies took into consideration only peak torque values and peak torque ratio of agonist to antagonists muscles at velocities $60 \%$ s and $180 \%$ s [4-10].

\section{Aim}

In this research we will evaluate peak torque, peak torque/body weight, total work, average power, range of motion and peak torque ratio of external to inter- 
nal rotators at 4 velocities $60 \%$ s, $120 \%$ s, $180 \%$ s and $240 \%$ to define functional disorders of shoulder joints in patients with rotator cuff injury. Moreover, we will try to correlate the above parameters with pain, disability and quality of life with the use of Shoulder Pain and Disability Index.

\section{Material and methods}

\section{Subjects}

Twenty subjects were evaluated. The first group consisted of ten patients with clinically diagnosed unilateral rotator cuff injury combined with shoulder impingement syndrome ( 5 women and 5 men). The average age of the patients was $59.7 \pm 17.94$ years, the average height was $168 \pm 8.92 \mathrm{~cm}$ and the average weight was $72.1 \pm 14.94 \mathrm{~kg}$. All of them had passed positively the followings tests: painful arc, Jobe's test, Neer and Kennedy-Hawkins impingement signs. Ultrasonography confirmed the narrowing of subacromial space and total disruption of supraspinatus muscle tendon. Exclusion criteria were: bilateral rotator cuff injury, previous injuries of shoulder joint, upper limb or neck instability, degenerative spine disease, rheumatoid arthritis. The symptoms were manifested from 6 to 12 months. Most subjects had applied some forms of physical therapy for their shoulder problem. It should be mentioned that we did not have any impact on the applied therapeutic method or any insight into details collected on the subject of the type of therapy provided. The second group consisted of 10 patients without shoulder pathology ( 4 women and 6 men). The mean age of the healthy control group was $25.9 \pm 3.81$ years, the mean height was $173.8 \pm 5.94 \mathrm{~cm}$ and the mean weight was $70.2 \pm 9.19 \mathrm{~kg}$. The age of the members in the control group was intentionally chosen to minimize the coexistence of any significant shoulder or cervical spine disorders typical for osteoarthritis in elderly people.

\section{Instruments}

Isokinetic testing of the shoulder rotator muscles was performed using a Biodex System 4 Pro Dynamometer. Measurements were performed in shoulder impingement group both on symptomatic and asymptomatic sides and in healthy volunteers on dominant and nondominant sides. The procedure used in the study was a bilateral concentric shoulder internal and external rotations protocol. Before testing subjects took part in a warming-up consisting of 5 minutes of upper limb exercises. Subjects were evaluated in the seated position with the arm in the plane of scapula, which is the shoulder position at $45^{\circ}$ abduction and at $30^{\circ}$ forward flexion. The elbow was at $90^{\circ}$ flexion and forearm was in the neutral position. Subjects' trunks were stabilized with seatbelts. The evaluation started with the external rotation. Isokinetic assessment was performed at 3 speeds: $120 \%$ s, $180 \%$ s and $240 \%$ s. The evaluation at each speed consisted of 5 repetitions. There was also an endurance trial, which included 10 repetitions at $240^{\circ} / \mathrm{s}$ velocity. A resting period of 30 seconds was kept between each run. During the test, the subjects were encouraged by the therapist to develop maximum strength in all contractions. Peak torque, peak TQ/BW, total work, average power, ROM and ERPT/IRPT ratio were assessed.

Pain and disability factors were evaluated with the numeric version of SPADI developed by Roach at 1991. It is consisted of two subscales: pain (5 questions) and disability (8 questions). The total SPADI score was counted up by averaging scores of these two subscales. The higher score represented greater intensity of pain and disability of shoulder joint.

\section{Statistical analysis}

All numerical data were expressed as mean \pm standard deviation values. Significant differences were evaluated using t-student test. Spearman's trial was used to assess correlations. The level of statistical significance was accepted at $p<0.05$. The results were analyzed using Statistica 10 software.

\section{Results}

Only in external rotation differences between symptomatic and asymptomatic sides were found. Table 1 shows the results of isokinetic external rotation test. In the values of peak torque from the patients' group there was found a significant discrepancy between symptomatic and asymptomatic side only at velocity $240 \%$ s. Similar differences were not found in the control group. When comparing deficits of peak torque between symptomatic and asymptomatic sides in patients' group and dominant and non-dominant side in the control group there was found a significant difference between deficit values at the highest tested velocity. It should be however underlined, that values of peak TQ/BW at all velocities significantly differed between symptomatic and asymptomatic sides what makes this parameter more sensitive than the peak torque alone. Moreover, no distinction between values of mentioned above parameter on dominant and non-dominant side was observed in the control group. 
There were not found any differences at average power referring to symptomatic and asymptomatic sides in the patients' group, dominant and non-dominant side in the control group or comparing deficits in the patients and control groups. The mean values of total work significantly differed between asymptomatic and symptomatic sides but not in the control group. The endurance test showed significant differences in values of peak TQ/BW and total work between symptomatic and asymptomatic side only in the patients' group.

Table 2 depicts results of ROM and ERPT/IRPT ratio. There were found differences between symptomatic and asymptomatic sides in the patients' group in ROM at each velocity. There was no contrast in this parameter in the control group. Comparing ERPT/IRPT ratio there were perceptible discrepancies between

Table 1. Isokinetic test results of external rotation. Significance accepted at $p<0.05$

\begin{tabular}{|c|c|c|c|c|c|c|c|}
\hline & Patients from g & $\begin{array}{l}\text { h shoulder imp } \\
\text { Irome }\end{array}$ & nent & \multicolumn{3}{|c|}{ Control group of healthy volunteers } & \multirow[t]{2}{*}{$p$} \\
\hline & \multicolumn{6}{|c|}{ Angular velocity $120 \% \mathrm{~s}$} & \\
\hline & asympt & sympt & $p$ & dom & nondom & $p p$ & \\
\hline Peak torque [Nm] & $20.7 \pm 7.7$ & $16.32 \pm 5.33$ & 0.16 & $19.7 \pm 6.9$ & $17.8 \pm 5.9$ & 0.53 & \\
\hline Deficit [\%] & \multicolumn{2}{|c|}{$18.05 \pm 20.53$} & \multicolumn{4}{|c|}{$7.6 \pm 15.5$} & 0.22 \\
\hline Peak TQ/BW [\%] & $28.1 \pm 5.1$ & $22.8 \pm 5.9$ & 0.04 & $27.6 \pm 8.05$ & $25.2 \pm 7.1$ & 0.48 & \\
\hline Avg. Power [W] & $20.4 \pm 11.4$ & $14.5 \pm 7.6$ & 0.19 & $23.2 \pm 10.0$ & $19.7 \pm 9.4$ & 0.43 & \\
\hline Deficit [\%] & \multicolumn{2}{|c|}{$25.0 \pm 29.8$} & \multicolumn{4}{|c|}{$15.1 \pm 15.2$} & 0.37 \\
\hline Total work [J] & $57.7 \pm 23.9$ & $36.9 \pm 18.0$ & 0.04 & $62.5 \pm 22.3$ & $52.0 \pm 23.0$ & 0.32 & \\
\hline \multirow[t]{3}{*}{ Deficit [\%] } & \multicolumn{2}{|c|}{$33.6 \pm 26.7$} & \multicolumn{4}{|c|}{$17.8 \pm 17.1$} & 0.13 \\
\hline & \multicolumn{6}{|c|}{ Angular velocity $180^{\circ} / \mathrm{s}$} & $p$ \\
\hline & asympt & sympt & $p$ & dom & nondom & $p p$ & \\
\hline Peak torque [Nm] & $20.9 \pm 6.8$ & $16.6 \pm 4.2$ & 0.11 & $17.7 \pm 6.2$ & $16.9 \pm 6.2$ & 0.78 & \\
\hline Deficit [\%] & \multicolumn{2}{|c|}{$17.5 \pm 17.2$} & \multicolumn{4}{|c|}{$3.6 \pm 21.7$} & 0.13 \\
\hline Peak TQ/BW [\%] & $28.7 \pm 5.0$ & $23.4 \pm 5.2$ & 0.03 & $24.9 \pm 7.1$ & $24.0 \pm 8.4$ & 0.8 & \\
\hline Avg. Power [W] & $21.5 \pm 12.6$ & $14.2 \pm 8.4$ & 0.15 & $22.5 \pm 10.7$ & $19.7 \pm 11.6$ & 0.58 & \\
\hline Deficit [\%] & \multicolumn{2}{|c|}{$34.1 \pm 14.4$} & \multicolumn{4}{|c|}{$15.4 \pm 27.5$} & 0.07 \\
\hline Total work [J] & $77.5 \pm 34.8$ & $48.5 \pm 22.0$ & 0.04 & $80.1 \pm 34.3$ & $67.1 \pm 41.8$ & 0.46 & \\
\hline \multirow[t]{3}{*}{ Deficit [\%] } & \multicolumn{2}{|c|}{$35.9 \pm 13.9$} & \multicolumn{4}{|c|}{$14.4 \pm 3$} & 0.05 \\
\hline & \multicolumn{6}{|c|}{ Angular velocity $240^{\circ} / \mathrm{s}$} & $p$ \\
\hline & asympt & sympt & $p$ & dom & nondom & $p p$ & \\
\hline Peak torque $[\mathrm{Nm}]$ & $21.7 \pm 7.0$ & $16.3 \pm 3.2$ & 0.04 & $15.8 \pm 6.0$ & $15.7 \pm 6.4$ & 0.98 & \\
\hline Deficit [\%] & \multicolumn{2}{|c|}{$19.9 \pm 22.8$} & \multicolumn{4}{|c|}{$0.61 \pm 14.2$} & 0.04 \\
\hline Peak TQ/BW [\%] & $30.3 \pm 7.4$ & $23.2 \pm 4.9$ & 0.02 & $22.4 \pm 8$ & $22.5 \pm 8.9$ & 0.99 & \\
\hline Avg. Power [W] & $17.1 \pm 7.7$ & $13.2 \pm 6.5$ & 0.24 & $18.3 \pm 9.0$ & $16.9 \pm 11.8$ & 0.77 & \\
\hline Deficit [\%] & \multicolumn{2}{|c|}{$19.4 \pm 29.1$} & \multicolumn{4}{|c|}{$16.9 \pm 28.5$} & 0.85 \\
\hline Total work [J] & $65.3 \pm 21.7$ & $45.0 \pm 16.2$ & 0.03 & $59.0 \pm 26.1$ & $55.4 \pm 35.3$ & 0.8 & \\
\hline \multirow[t]{3}{*}{ Deficit [\%] } & \multicolumn{2}{|c|}{$29.0 \pm 20.0$} & \multicolumn{4}{|c|}{$15.1 \pm 26.3$} & 0.2 \\
\hline & \multicolumn{6}{|c|}{ Angular velocity $240 \%$ s (endurance) } & $p$ \\
\hline & asympt & sympt & $p$ & dom & nondom & $p p$ & \\
\hline Peak torque $[\mathrm{Nm}]$ & $19.3 \pm 5.5$ & $16.1 \pm 3.5$ & 0.14 & $17.1 \pm 6.05$ & $16.9 \pm 7.39$ & 0.95 & \\
\hline Deficit [\%] & & & & & & & 0.19 \\
\hline Peak TQ/BW [\%] & $26.8 \pm 5.0$ & $22.7 \pm 4.8$ & 0.08 & $24.4 \pm 8.1$ & $24.3 \pm 10.9$ & 0.99 & \\
\hline Avg. Power [W] & $18.4 \pm 9.9$ & $13.9 \pm 8.1$ & 0.29 & $19.4 \pm 10.5$ & $16.1 \pm 11.2$ & 0.51 & \\
\hline Deficit [\%] & & & & & & & 0.87 \\
\hline Total work [J] & $123.4 \pm 41.2$ & $90.8 \pm 38.6$ & 0.09 & $123.3 \pm 60.1$ & $105.7 \pm 72.0$ & 0.56 & \\
\hline Deficit [\%] & & & & & & & 0.81 \\
\hline
\end{tabular}

sympt - symptomatic side; asympt-symptomatic side; dom - dominant side; nondom - nondominant side

Deficit:

1 to $10 \%$ - No significant difference between extremities

11 to $25 \%$ - Rehabilitation recommended to improve muscle performance balance

> 25\% - Significant Functional Impairment

(-) Negative deficit indicates that a symptomatic extremity performed better than asymptomatic one 
Table 2. Isokinetic tests results: ROM and External rotation peak torque (ERPT)/Internal rotation peak torque (IRPT)

\begin{tabular}{|c|c|c|c|c|c|c|}
\hline & \multicolumn{3}{|c|}{ Patients from group with shoulder impingement syndrome } & \multicolumn{3}{|c|}{ Control group of healthy volunteers } \\
\hline & \multicolumn{6}{|c|}{ Angular velocity $120 \%$} \\
\hline & asympt & sympt & $p$ & dom & nondom & $\mathrm{p}$ \\
\hline ROM & $89.17 \pm 6.17$ & $76.63 \pm 14.09$ & 0.02 & $93.26 \pm 11.48$ & $86.31 \pm 8.93$ & 0.15 \\
\hline \multirow[t]{3}{*}{ ERPT/IRPT } & $68.68 \pm 17.53$ & $58 \pm 9.27$ & 0.13 & $69.12 \pm 13.02$ & $66.47 \pm 16.09$ & 0.69 \\
\hline & \multicolumn{6}{|c|}{ Angular velocity $180 \% \mathrm{~s}$} \\
\hline & asympt & sympt & $p$ & dom & nondom & $p$ \\
\hline ROM & $88.65 \pm 6.17$ & $76.42 \pm 13.11$ & 0.02 & $92.9 \pm 11.3$ & $86.55 \pm 9.17$ & 0.19 \\
\hline \multirow[t]{3}{*}{ ERPT/IRPT } & $70.09 \pm 14.5$ & $60.6 \pm 8.77$ & 0.09 & $69.64 \pm 20.23$ & $69.72 \pm 22.94$ & 0.99 \\
\hline & \multicolumn{6}{|c|}{ Angular velocity $240 \% / s$} \\
\hline & asympt & sympt & $p$ & dom & nondom & $p$ \\
\hline ROM & $87.78 \pm 6.06$ & $75.57 \pm 12.93$ & 0.01 & $92.01 \pm 11.15$ & $85.89 \pm 9.98$ & 0.21 \\
\hline \multirow[t]{3}{*}{ ERPT/IRPT } & $85.02 \pm 17.96$ & $69.71 \pm 9.73$ & 0.03 & $73.45 \pm 24.88$ & $80.04 \pm 28.23$ & 0.59 \\
\hline & \multicolumn{6}{|c|}{ Angular velocity $240 \%$ s (endurance) } \\
\hline & asympt & sympt & $p$ & dom & nondom & $p$ \\
\hline ROM & $87.96 \pm 6.13$ & $75.64 \pm 13.11$ & 0.01 & $92.16 \pm 11.29$ & $85 \pm 9.79$ & 0.19 \\
\hline ERPT/IRPT & $67.57 \pm 11.29$ & $53.55 \pm 8.37$ & 0.01 & $70.98 \pm 20.51$ & $74.81 \pm 26.94$ & 0.72 \\
\hline
\end{tabular}

symptomatic and asymptomatic side at velocity $240 \% \mathrm{~s}$ and during the endurance trial. There was not any difference in the control group.

Table 3 shows results of SPADI test. Statistical analysis showed significant differences between symptomatic and asymptomatic sides in results of SPADI questionnaire, but there was no correlation found between SPADI and isokinetic evaluation.

Table 3. Shoulder Pain and Disability Index

\begin{tabular}{lccc}
\hline & sympt & asympt & $p$ \\
\hline Pain & 0.41 & 0 & 0.004 \\
\hline Disability & 0.21 & 0 & 0.002 \\
\hline Total & 0.34 & 0 & 0.003 \\
\hline
\end{tabular}

\section{Discussion}

The results of the present study demonstrated a decrease in functionality of patients with shoulders impingement syndrome in isokinetic assessment as well as in pain and disability evaluation. In the patients' group, deficits of external rotators were present while internal rotators didn't exhibit significant weaknesses. The significant differences were observed in mean values of peak TQ/BW, total work, ROM and SPADI evaluation. There were deficits in peak torque ERPT/ IRPT ratio, but only at $240 \%$ s velocity. No significant deficits between dominant and non-dominant sides in the control group were noticed. Variations of mean peak torque values were found only at $240 \%$, when comparing both symptomatic and asymptomatic sides in the patients' and control group. It is possible that deficits appear only at higher velocities. This might be explained by morphologic changes in the muscle tissue. Irlenbush and Gansen performed a biopsy of supraspinatus muscle and the acromial part of deltoid muscle in patients with a supraspinatus syndrome and partial or complete supraspinatus rapture. This research demonstrated abnormal fibers distribution in those muscles. Their study highlighted greater changes at fast-twitch fibers (type II), which are rapidly contracting elements but undergo the quick fatigue, than slow-twitch fibers (type I), which are slowly contracting structures but they are fatigue resistant [11]. This is the explanation of the deficit observed in our study at higher velocities of testing.

Previous studies described measurements of peak torque at lower velocities: $60^{\circ} \% \mathrm{~s}$ and $180^{\circ} / \mathrm{s}$. Erol et al compared the mean peak torque values of patients with subacromial impingement syndrome and in a control group. It should be mentioned that only velocity of $60 \%$ was considered. Additionally, only the significant deficit of internal rotators compared in patients and a control group was detected. In the control group, the mean value of peak torque measured on the dominant side was significantly higher than on non-dominant [4]. Tyler et al tested isokinetically the peak torque at $60 \%$ and $180 \%$ velocities. They compared patients with subacromial impingement syndrome with control group, but didn't find any major deficits of peak torque in these velocities [5]. Also, the position of shoulder and range of motion may have impacted the results. We were testing shoulders in a scapular plane and the painless range of motion. Scapular plane position guaranteed a comfortable, physiological position, which ensured the optimum result of peak torque measure- 
ments. It didn't cause any symptoms of impingement, as it didn't exert the pressure on tissues. Moreover, the peak torque was measured at the middle of range of motion in isokinetic tests but the impingement occurs only when the shoulder is at the end of range. So, both position and range of motion considered in this study, didn't cause symptoms of impingement. Tyler et al tested rotator cuff power isokinetically in two different positions, and with a handheld dynamometer (HHD) as an isometric strength trial. Testing positions were scapular plane and shoulder abduction to $90^{\circ}$ in a frontal plane. They didn't find any significant deficits in isokinetic tests, but testing in shoulder abduction positions demonstrated higher deficits of peak torque. Using HHD testing they found deficits both at scapular plane and shoulder abductions positions. They indicated that testing using HHD may be more sensitive than isokinetic testing alone, because HHD evaluates strength at the end of a range. The authors believe that strength may be normal at the middle range, but deficits may appear at the end of range, when there can appear the symptoms of impingement [5]. Dupuis et al didn't find any significant differences between results of scapular plane testing and shoulder abduction testing. However, they tested only healthy people who didn't show symptoms of shoulder impigement. No pain appeared while testing this range of motion, which could affect final results [6]. We didn't find previous studies describing isokinetic testing at $240 \%$ s velocities. In our study deficits of peak torque values have already appeared only at $240 \%$ s. The findings of our research provide the evidence that peak torque should be tested only at such a velocity.

We detected significant differences between mean values of ERPT/IRPT ratio only at $240 \%$ s. The same phenomenon was detected during the endurance trial. It should be underlined that this was calculated for the peak torque values. That is why a relation between results of peak torque and ERPT/IRPT ratio may occur. Erol et al compared results of mean values ERPT/IRPT ascertained in patients with results obtained in a control group. Results were similar. This might indicate that both external rotators and internal rotators were impaired [4]. Mattielo-Rosa et al compared results of ERPT/IRPT measurements in patients and a control group at $60 \%$ s and $180 \%$ s velocities. They found significant differences of this ratio only at higher velocity [10]. Edouard et al indicated that peak torque is more reliable than ERPT/IRPT, because it has an impact on the two values of this ratio thus, a distortion of each of them may change the result [7].
Peak TQ/BW seems to be more reliable indicator than peak torque, because it takes the anthropometric attributes of a subject into consideration. In our study, symptomatic upper limb gained significant higher result than asymptomatic one at $120 \%$ s, $180 \%$ s and $240 \%$ s. Unfortunately, there are only few studies describing changes of this parameter. Wassinger et al tested peak TQ/BW at $60 \%$ and observed significant weakness of symptomatic side in comparison with asymptomatic one [8]. In our study a value of peak $\mathrm{TQ} / \mathrm{BW}$ was lowered in symptomatic extremity even at $60 \%$ s and $180 \%$ s velocities. We believe that this is better factor to evaluate dysfunction of a rotator cuff muscle in comparison with peak torque. Mean values of both total work and ROM demonstrated dysfunction of shoulder joint. No mention of these factors was found in a previous literature. Mean values of ROM are often evaluated during shoulder joint functionality trial, but none of them tested this factor during isokinetic condition. Deficits of total work mean values appeared even at lower velocities than $240 \%$ s. It seems to be a reliable factor through which an evaluation of functions of shoulder joints can be performed.

We also found significant differences between mean values of SPADI test referring to symptomatic and asymptomatic sides. Additionally, there were no correlations between the result of isokinetic assessment and SPADI test. As it has been already mentioned, during the biodex test patients didn't feel any pain and that is why there was no correlation between those two tests. Erol et al provided the same explanation of lack of relation between results of these two trials. In addition, they think that low velocities may have an impact on the results of this test [4]. Wessinger et al evaluated influence of pain on muscle strength in isokinetic assessment. The authors compared mean peak torque values with mean value of VAS results before and after analgesic injection in the subacromial space. Their study showed that the level of pain affected the result of strength evaluation. Their patients demonstrated weakness both in external and internal rotations. After a decrease of pain, mean strength values of symptomatic side came closer to the results detected on the asymptomatic side. Mean value of peak TQ/BW during external rotation increased, but it was still lower than strength found on asymptomatic side. Pain had a large impact on a decrease of strength in patients with rotator cuff disease, particularly with partial thickness tearing and inflammation [8]. Forthomme et al considered the impact of pain levels on the mean peak torque value. They observed that after analgesic injection only 
external rotators were still weakened. It might indicate that external rotators were more affected than internal ones [9].

Psychological factors may have an impact on the lack of correlation between results of SPADI test and isokinetic assessment. It has to be highlighted that the pain is a subjective sensation and patient's personality may affect the result of test. Hill et al evaluated the impact of range of motion in shoulder joint on result of SPADI test. They also didn't find any correlation [12]. There are many studies which acknowledge the reliability of SPADI questionnaire [13-15].

\section{Conclusions}

The results of this study showed a significant decrease of external rotators function expressed in mean values of peak torque, peak TQ/BW and total work. Additionally, the abnormal relationships between agonistic and antagonistic muscles strength and decrease of ROM were discovered in patients representing the shoulder impingement syndrome. Decrease of shoulder joint functionality detected in isokinetic evaluation was confirmed in results of SPADI test. Moreover, the results indicate that higher velocities of isokinetic testing are more reliable for evaluation of shoulder joint.

\section{Acknowledgements}

\section{Conflict of interest statement}

The authors declare that there is no conflict of interest in the authorship or publication of contribution.

\section{Funding sources}

There are no sources of funding to declare.

\section{References}

1. Clement ND, Nie YX, McBirnie JM. Management of degenerative rotator cuff tears: a review and treatment strategy. Sports Med Arthrosc Rehabil. Ther Technol. 2012;4:48.

2. Robin MS, Andrew LW. Degenerative rotator cuff disease and impingement. Orthop Traum. 2011;25(1):1-10.

3. Umer M, Qadir I, Azam M. Subacromial impingement syndrome. Orthop Rev. 2012;4(2): e18.

4. Erol O, Ozcakar L, Celiker R. Shoulder rotator strength in patients with stage I-II subacromial impingement: Relationship to pain, disability, and quality of life. J Should Elb Surg. 2008;17(6):893-897.

5. Tyler TF, Nahow RC, Nicholas SJ, McHugh MP. Quantifying shoulder rotation weakness in patients with shoulder impingeent. J Should Elb Surg. 2005;14:570-574.
6. Dupuis C, Chollet CT, Leroy D, Blanquart FB. Influences the position of the scapula in isokinetic assessment: An example with high level athletes. Isokinet Exerc Sci. 2005;13:63-66.

7. Edouard $P$, Codine $P$, Samozino $P$, Bernard $P L$, Hérisson C, Gremeaux V. Reliability of shoulder rotators isokinetic strength imbalance measured using the Biodex dynamometer. J Sci Med Sport. 2013;16(2):162-165.

8. Wassinger CA, Sole G, Osborne $\mathrm{H}$. The role of experimentally-induced subacromial pain on shoulder strength and throwing accuracy. Man Ther. 2012;17:411-415.

9. Forthomme B, Croisier JL, Huskin JP, Crielaard JM. Isokinetic assessment of shoulders with impingement syndrome following pain inhibition. Isokinet Exerc Sci. 2003; 11:70-71.

10. Mattiello-Rosa SM, Camargo PR, Santos AA, Pádua M, Reiff RB, Salvini TF. Abnormal isokinetic time-to-peak torque of the medial rotators of the shoulder in subjects with impingement syndrome. J Should Elb Surg. 2008; 17(1):54-60.

11. Irlenbusch U, Gansen HK. Muscle biopsy investigations on neuromuscular insufficiency of the rotator cuff: a contribution to the functional impingement of the shoulder joint. J Should Elb Surg. 2003;12(5):422-426.

12. Hill CL, Lester S, Taylor AW, Shanahan ME, Gill TK. Factor structure and validity of the shoulder pain and disabilty index in a population - based study of people with shoulder symptoms. BMC Musculoskel Disord. 2011;12:8.

13. MacDermid JC, Solomon P, Prkachin K. The Shoulder Pain and Disability Index demonstrates factor, construct and longitudinal validity. BMC Musculoskel Disord. 2006;7:12.

14. Ekeberg OM, Bautz-Holter E, Tveita EK, Keller A, Juel NG, Bronx JI. Agreement, reliability and validity in 3 shoulder questionnaires in patients with rotator cuff disease. BMC Musculoskel Disord. 2008;9:68.

15. Staples MP, Forbes A, Green S, Buchbinder R. Shoulder-specific disability measures showed acceptable construct validity and responsiveness. J Clin Epidemiol. 2010; 63:163-170.

Acceptance for editing: 2014-12-10 Acceptance for publication: 2014-12-31

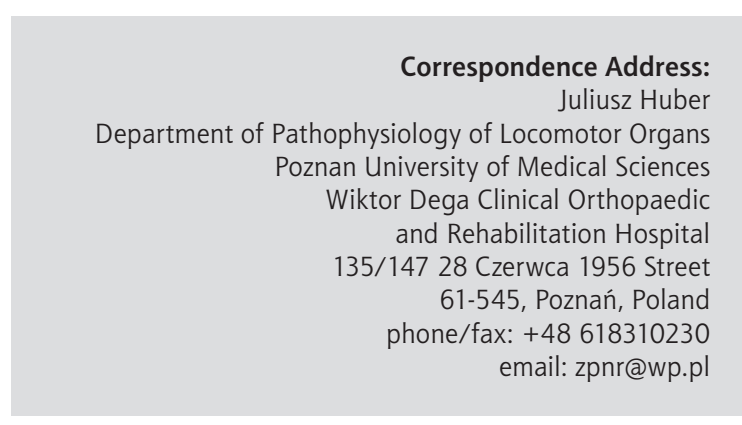

